\title{
Ludomática en ambientes de aprendizaje: educación rural en el posconflicto colombiano
}

\section{Diego Armando Bautista Díaz}

https://orcid.org/oooo-0001-5159-7853 Corporación Universitaria Minuto de Dios, Colombia

dbautista@redacademica.edu.co

\section{Eurias Casas Casallas}

https://orcid.org/oooo-0002-4872-2059 Corporación Universitaria Minuto de Dios, Colombia

eurias.casas@uniminuto.edu

\section{Zaily del Pilar García Gutiérrez}

https://orcid.org/0ooo-0002-9846-7762 Corporación Universitaria Minuto de Dios, Colombia zgarcia@uniminuto.edu

\section{Jhonny Gómez Amaya}

https://orcid.org/oooo-0oo1-9323-337X Corporación Universitaria Minuto de Dios, Colombia jhonny.gomez@uniminuto.edu

\section{Bladimir Alexander Gutiérrez Castro}

https://orcid.org/oooo-ooo1-676o-3736 Corporación Universitaria Minuto de Dios, Colombia bgutierrez@uniminuto.edu

\section{Resumen}

El artículo propone una estrategia pedagógica para el fomento de la educación mediática, desde la lúdica y la mediación de tecnologías, para la resignificación del conflicto armado colombiano, vinculando a la comunidad educativa. El proceso pedagógico se desarrolló en el marco metodológico de la acción participativa, en dos instituciones educativas públicas con sus extensiones rurales de la región del Gualiva en Nimaima, Cundinamarca (Colombia). La intervención se realizó durante dos años, empezando por caracterizar recursos físicos y conocimientos tecnológicos de la comunidad, como base para formular la estrategia pedagógica en competencias mediáticas. Asímismo, se desarrolló y validó un ambiente virtual de aprendizaje (AVA) y un aplicativo móvil (APP). Se identificó que la implementación de estrategias educativas mediadas por tecnologías de la información y la comunicación (TIC) aporta a la disminución de las brechas digitales, mejora procesos comunicativos y permite la reconfiguración de las dinámicas del territorio.

\section{Palabras clave (Fuente: tesauro de la Unesco)}

Colombia; conflicto armado; educación de la primera infancia; educación rural; guerra; población rural. 


\title{
Ludomática in Learning Environments: Rural Education in the Colombian Post-conflict
}

\begin{abstract}
This article proposes a pedagogical strategy for promoting media education, using games and technology mediation, in order to resignify the Colombian armed conflict and involve the educational community. The pedagogical process was conducted within the methodological framework of participatory action, at two public schools and their rural extensions in the Gualiva region in Nimaima, Cundinamarca (Colombia). The intervention lasted two years and began by characterizing the community's physical resources and technological knowledge as a basis for formulating a pedagogical strategy for media literacy. A virtual learning environment (VLE) and a mobile application (APP) were also developed and validated. It was identified that the implementation of educational strategies mediated by information and communication technologies (ICT) reduces digital gaps, improves communication processes, and reshapes territorial dynamics.
\end{abstract}

\section{Keywords (Source: Unesco Thesaurus)}

Colombia; armed conflict; early childhood education; rural education, war; rural population. 


\title{
Ludomática em ambientes de aprendizagem: educação rural no pós-conflito colombiano
}

\author{
Resumo
}

Este artigo propõe uma estratégia pedagógica para o fomento da educação midiática, a partir da ludicidade e da mediação de tecnologias, para ressignificar o conflito armado colombiano, vinculando a comunidade educativa. O processo pedagógico foi desenvolvido no referencial metodológico da ação participativa, em duas instituições educativas públicas com suas extensões rurais da região do Gualiva em $\mathrm{Ni}$ maima, Cundinamarca (Colômbia). A intervenção foi realizada durante dois anos, começando por caracterizar recursos físicos e conhecimentos tecnológicos da comunidade, como base para formular a estratégia pedagógica em competências midiáticas. Além disso, foram desenvolvidos e validados um ambiente virtual de aprendizagem (AVA) e um aplicativo móvel (APP). Foi identificado que a implantação de estratégias educativas mediadas por tecnologias da informação e da comunicação contribui para diminuir as lacunas digitais, melhorar processos comunicativos e permite a reconfiguração das dinâmicas do território.

\section{Palavras-chave (Fonte: Tesauro da Unesco)}

Colômbia; conflito armado; educação infantil; educação rural; guerra; população rural. 


\section{Introducción}

La condición actual de la sociedad del conocimiento exige a los gobiernos de cada país y específicamente a las instituciones educativas hacer esfuerzos para que su población sea eficiente en el manejo de las herramientas tecnológicas e informacionales. En consecuencia, es urgente diseñar y poner en marcha propuestas pedagógicas que fortalezcan las competencias básicas para el manejo de las tecnologías de la información y la comunicación (TIC). Este documento da cuenta de los resultados de una investigación que se adelantó en el municipio de Nimaima, en Cundinamarca (Colombia), con la que se pretendió fortalecer las competencias en el manejo de las herramientas tecnológicas e informacionales en niños que asisten a las escuelas y que, para el caso, tienen antecedentes de victimización por el fenómeno de la guerra.

Colombia es un país que se destaca por su diversidad y riqueza cultural: la creatividad, el emprendimiento y la capacidad de superación son una constante. También se caracteriza por tener una historia marcada por el conflicto armado que, inicialmente, se planteó como alternativa para superar la inequidad social, después se resignificó como un foco de injusticia, inseguridad y muerte y ahora atraviesa por un proceso de paz. Este tipo de contrastes hacen de la educación una herramienta de mediación para potenciar las fortalezas culturales y superar el conflicto. Con esta perspectiva surgió la investigación "Ambientes de aprendizaje desde la ludomática para el desarrollo de la comunicación y la tecnología de los niños en el marco del posconflicto", como una propuesta alternativa de educación para las infancias basada en el uso de TIC, la lúdica, la participación y el diálogo, con la intención de aportar en la construcción de prácticas más equitativas y el desarrollo de la comunidad.

Este proyecto se llevó a cabo en el municipio de Nimaima, ubicado en la provincia del Gualivá, a 75 km de Bogotá. La actividad económica más destacada en dicha población es la agricultura: se cultiva la caña de azúcar, cuyo derivado es la panela. Además, como consecuencia de la variedad de fauna y flora y por su riqueza hídrica, en las últimas décadas el pueblo se ha convertido en un escenario para el ecoturismo y la práctica de deportes extremos. En contraste con lo anterior, Nimaima se ha catalogado como uno de los sectores afectados por el conflicto armado en el país, aunque en la actualidad se encuentra en un proceso de reconciliación gracias a que sus habitantes están comprometidos con la transformación de su territorio. El pueblo cuenta con 3222 habitantes, según el informe del Sistema de Selección de Beneficiarios para Programas Sociales (DNP, 2018). De modo específico, se trabajó con dos sedes educativas departamentales: Cacique Anamay, ubicada en el casco urbano del pueblo, y Misael Pastrana (con sus respectivas extensiones rurales), ubicada en la Inspección de Tobia.

Los participantes del proyecto hacen parte de una comunidad rural, cuya fuerza laboral está centrada en las tareas agrícolas y las labores del hogar. Estas personas poseen bajo nivel de formación académica, poco interés por las innovaciones y los avances tecnológicos y mínimas posibilidades de acceso y uso de internet. Esto último afecta los procesos de enseñanza-aprendizaje de los niños, por lo cual es fundamental llevar a cabo iniciativas que aporten a la transformación gradual de esta situación. Además, los niños de esta población no alcanzan los aprendizajes esperados de su nivel de formación, lo cual se evidencia en las escasas competencias frente a lo esperado para el grado escolar que cursan.

La situación descrita hasta el momento conlleva un análisis profundo de las diversas acciones que el sistema educativo colombiano debe implementar para mitigar el atraso y las brechas educacionales que la violencia ha dejado, particularmente, en la población infantil. En el caso de Nimaima, existe una necesidad sentida de generar estrategias de enseñanza que permitan a los maestros contar con herramientas y mediaciones didácticas de apoyo a la formación integral de las infancias. Por ello 
es menester realizar esfuerzos para acercar a esta población herramientas tecnológicas como aporte al desarrollo y/o fortalecimiento de competencias relacionadas con las dinámicas y escenarios de la virtualidad, en tanto constituyen habilidades que demanda la sociedad actual y sus contextos, de conformidad con el actuar ciudadano y el desenvolvimiento profesional, en el marco de la globalización y la apuesta por la alfabetización en tecnología. Esta problemática lleva a la formulación de la siguiente pregunta: ¿cómo se fortalece la formación de las infancias desde los ambientes virtuales de aprendizaje, ante los retos del posconflicto? Desde esta perspectiva, la investigación profundiza conceptualmente acerca de los retos que presenta el posconflicto, la educación mediática, los ambientes de aprendizaje y la educación de las infancias.

En términos aplicados, la investigación aporta el desarrollo de una estrategia pedagógica mediada por el uso de tecnologías y pensada para la reconstrucción del territorio y la resignificación del conflicto. Centrada en la formación de los niños, a su vez vincula a la comunidad educativa como un aporte a la consolidación de escenarios de paz y la mejora de los escenarios escolares. Se da por entendido que el acceso a la educación es una herramienta muy importante para la protección de los niños y también para la comunidad en general (Infante, 2011).

Finalmente, luego de pasar revista a los análisis y estudios sobre educación para el conflicto, se establece que el reto consiste en desarrollar tareas de protección y desarrollo, orientadas especialmente a las personas escolarizadas, que para el caso de esta investigación son niños de educación básica primaria del sector rural, para que se pueda controlar o evitar la vulnerabilidad de los sujetos frente a los actores que propician la violación de los derechos (Pérez, 2014); por otro lado, se propone que otra tarea indispensable es proveer una estructura, un propósito y un significado cotidiano para los niños y los adolescentes, puesto que se debe garantizar que la escuela no sufra los impactos del conflicto, ya que el papel de la educación es constituir procesos de mediación, vitales para la reconciliación, la resignificación, la justicia y la superación de la guerra.

\section{El rol de la educación en contextos de posconflicto}

Abordar el posconflicto requiere definir qué es el conflicto. Este hace referencia "a la violencia armada en la que se enfrentan grupos de varias índoles, tales como fuerzas militares, guerrillas, grupos armados, paramilitares, comunidades religiosas o étnicas, empleando armas u otros métodos destructivos" (Fride, 2006, p. 2). Según esto, Colombia ha vivido su historia inmersa en el conflicto armado, el cual ha sido más agudo en los espacios rurales que en los urbanos, en sus causas y en sus consecuencias.

En el mismo sentido, Berdal y Malone (2000) afirman que las causas del conflicto tienden a sobreponer los aspectos sociopolíticos. Collier et al. (2003), por su parte, las basan en problemas de identidad territorial. Lo primero obedece a razones de índole social -específicamente, de orden económico- en relación con la agudización de la pobreza, la inequidad y la desigualdad de los sectores urbanos y rurales más desfavorecidos, más aún cuando el discurso sociopolítico se ha encargado de perpetuar estas prácticas de discriminación en el devenir histórico, sin llegar a soluciones efectivas.

En cuanto a la segunda causa, comporta el no reconocimiento de una identidad territorial e histórica, definida bajo el carácter subjetivo de los sentimientos de pertenencia colectiva a un espacio territorial, cultural, histórico, económico y diverso. La pérdida de identidad por la desapropiación del territorio por medios violentos, la colonización o la expropiación conlleva una lucha justificada entre aquellos que creen ser los herederos y aquellos que afirman ser los nuevos propietarios producto del desplazamiento. Por ende, se puede afirmar que la identidad es "base fundamental del resurgimiento de un conflicto, debido al papel que juega en la razón de ser y estar, en los seres humanos" (Filipov, 2008, p. 17). 
Con respecto al concepto de posconflicto, Collier et al. (2003), Jiménez, De Currea-Lugo y Grabe (2008) y Berdal y Malone (2000) concuerdan en que no existe una definición oficial del mismo. Sin embargo, la Fundación para las Relaciones Internacionales y el Diálogo Exterior (Fride, 2006) lo considera un periodo de tiempo en el que los conflictos violentos pasados se han minimizado lo suficiente como para que inicien las actividades de reintegración y rehabilitación. En otras palabras, se determina como un "después esperanzador", en el cual se debe restituir a las víctimas del conflicto en dimensiones como salud, vivienda y educación (destacándose lo socioeconómico), entre otras.

Como punto de partida para evitar el resurgimiento de un conflicto es fundamental aportar a la creación de un sistema educativo sólido que entienda y oriente los procesos necesarios para abolir el conflicto. Así, la educación ayuda a reducir polarizaciones económicas, sociales y étnicas, a la vez que se convierte en una institución que aporta al crecimiento y desarrollo equitativo en el territorio con el fin de construir una cultura de no violencia.

En coherencia con lo anterior, y en pro de la construcción de estrategias educativas que impacten positivamente a la población en Nimaima en el marco del posconflicto, se presenta el diseño de una propuesta formativa para los niños y niñas, basada en los aspectos enunciados por Garzón (2003):

- $\quad$ Contexto: cada territorio tiene sus propias características de rehabilitación y construcción. Se denomina territorio a todo espacio físico afectado en su legitimidad, operatividad, seguridad y pertenencia por el conflicto armado.

- Actores: constituyen el sujeto principal de la etapa de posconflicto. Hacia ellos se dirige la mayor parte del proceso de rehabilitación y construcción.

- Rehabilitación institucional: implica repensar el papel de participación del Estado y acciones, políticas y leyes pertinentes a la situación actual y futura del territorio en posconflicto.

- $\quad$ Anticipación del problema: realización de planes estratégicos, previniendo situaciones adversas e imprevistos.

- $\quad$ Avance: alternativas de cambio y actualización en todas las dimensiones (cultural, social, educativa y económica, entre otras.)

- Asistencia: planes de atención humanitaria de corto y largo plazos. Al igual que el conflicto, la rehabilitación también es duradera.

En conclusión, el diseño de una propuesta pedagógica en el marco del posconflicto contribuye al mejoramiento continuo de una nueva sociedad; es más, resulta la base fundamental de su desarrollo, ya que la educación se configura como pilar esencial para mediar la superación de los conflictos o crisis (Nicolai y Triplehorn, 2003).

En esta dirección, no se puede desconocer que existe una amplia gama de propuestas pedagógicas, algunas más pertinentes que otras, ante los retos del posconflicto. Por ello, esta investigación optó por explorar una alternativa basada en la educación mediática, en tanto esta busca fortalecer las competencias mediáticas pertinentes para la formación de ciudadanos competentes.

\section{Educación mediática y uso de herramientas tecnológicas}

Son varios los términos que se han utilizado durante las últimas décadas que hacen referencia a la formación de ciudadanos competentes en el manejo de las herramientas informacionales y tecnológicas; por ejemplo, educación en medios, alfabetización mediática, alfabetización digital, educación mediáti$\mathrm{ca}$, entre otros. Asimismo, son diversos los recursos tecnológicos que se han construido y que, sin lugar a dudas, "han transformado la forma de comunicar y han modificado la información" (Daskal, 2018, p. 241). En ese sentido, es urgente también transformar los 
actos educativos con la intención de que estos respondan a las exigencias de la actual sociedad de la información (Pounaki, Givi y Fahimnia, 2017).

Partiendo de las ideas anteriores, y en coherencia con los propósitos y la perspectiva que estructura esta investigación, se incorpora el concepto de educación mediática, como aquella que:

Comporta el dominio de conocimientos, destrezas y actitudes relacionados con seis dimensiones básicas 1) lenguajes, 2) tecnología, 3) procesos de interacción, 4) procesos de producción y difusión, 5) ideología y valores y 6) estética. Estos indicadores tienen que ver, según los casos, con el ámbito de participación como personas que reciben mensajes e interaccionan con ellos (ámbito del análisis) y como personas que producen mensajes (ámbito de la expresión). (Ferrés y Piscitelli, 2012, p. 79)

Dicho de otro modo, quien cuenta con competencias mediáticas tiene conocimientos y destrezas en el análisis y la expresión de mensajes. Para esta investigación solo se busca fortalecer tres dimensiones, por cuanto son las que más se ajustan a las características de los participantes: tecnología; procesos de interacción; y producción y difusión. Estas dimensiones son las que permiten a los niños, a partir de la mediación tecnológica, fortalecer las competencias en el uso y la interacción con la tecnología y en la producción y difusión de mensajes.

\section{Propuesta de diseño para ambientes virtuales de aprendizaje (AVA)}

Una propuesta de ambientes virtuales de aprendizaje (AVA) debe tener en cuenta la articulación entre procesos individuales y sociales de los niños al momento de aprender, ya que son la base para diseñar un entorno de aprendizaje que potencie el diálogo y la participación de los involucrados. Desde la perspectiva de esta investigación, se trata de los AVA requeridos en la comunidad que adelanta un proceso de posconflicto. Asimismo, debe tenerse en cuenta la coherencia entre el modelo pedagógico, los usuarios -según los roles de docentes y estudiantesy las posibilidades de la tecnología (Salinas, 2004). Seguidamente, se exponen los elementos básicos que permiten reconocer las potencialidades de uso de herramientas en el diseño de ambientes virtuales de aprendizaje.

En esta investigación se tomaron como referencia el diseño instruccional ADDIE: análisis, diseño, desarrollo, implementación y evaluación (Belloch, 2013). Los elementos que orienta el diseño instruccional son:

- Identificación del sujeto como centro del proceso de enseñanza-aprendizaje.

- Definición de objetivos de la instrucción dirigidos a la adquisición de conocimiento desde una oportunidad práctica de exploración.

- Análisis del contexto y del sujeto que permita configurar las acciones de formación acordes a las necesidades detectadas.

- $\quad$ Planeación de las acciones de enseñanza y aprendizaje de manera flexible, pero sistemática.

- Puesta en marcha de los elementos planeados en un proceso de constante revisión de aciertos o fallas.

- Evaluación como proceso permanente en el desarrollo de la instrucción.

- Reflexión sobre los alcances del proceso adelantado, con la intención de aportar a la transformación.

- Identificación del potencial de los recursos tecnológicos para favorecer la reflexión sobre el propio proceso.

De manera que el diseño de un AVA, como acto educativo y pedagógico, debe responder los siguien- 
tes cuestionamientos: a quién está dirigido, con qué objetivo(s) y alcance(s) y a través de qué medio o medios. También debe tener en cuenta la población, el contexto, los propósitos e intenciones y, por último, el conjunto de recursos, contenidos y actividades. A propósito de ello, Herrera sugiere que "un ambiente de aprendizaje constituye un espacio propicio para que los estudiantes obtengan recursos informativos y medios didácticos para interactuar y realizar actividades encaminadas a metas y propósitos educativos previamente establecidos" (2006, p. 2). Esta premisa fue el punto de partida para diseñar un AVA dirigido a niños cuyas edades oscilan entre los 6 y 12 años, pertenecientes a los grados tercero a quinto de primaria de las instituciones educativas de Nimaima.

\section{Educación infantil, retos de formación en ambientes de aprendizaje para la educación mediática}

La creación de un AVA como propuesta de formación de educación infantil -en este caso, desde los postulados de la educación mediática- debe partir de establecer claramente la postura epistemológica desde la cual se explica la forma en que los niños aprenden, por cuanto la comprensión que se tenga de este fenómeno causará alteraciones en las estrategias de enseñanza en las que se soporta la propuesta formativa. Para el caso de esta investigación, se plantea que los niños son seres integrales, pues experimentan un crecimiento social y cognitivo conjunto y armónico (Lillard et al., 2017). Desde esta perspectiva, el aprendizaje se configura como una práctica vital y se constituye así en un acto complejo y sistémico con el cual se supera el reduccionismo y la fragmentación del ser. En palabras de Morin, esto corresponde a definir que el organismo "significa totalidad organizada, pero de un tipo diferente de máquina artificial, ya que la alternativa de reduccionismo no está en un principio vital, sino en una realidad organizada y viviente" (2009, p. 52). Así, entonces, el acto de aprender es un proceso inherente a la vida humana (Sandberg et al., 2017), por lo cual se puede afirmar que, desde la gestación, se cuenta con una predisposición biológica para desarrollar conocimientos y aprendizajes de acuerdo con el proceso evolutivo, madurativo y sociocultural de los niños. Desde esta perspectiva se propone que el aprendizaje es un proceso psicogenético asociado a la maduración y la actividad del sujeto, en la medida en que tiene la oportunidad de manipular, experimentar y apropiar el objeto inicialmente concreto y luego abstracto, experiencias que se configuran como aspectos determinantes del proceso de desarrollo y aprendizaje (Antoranz y Villalba, 2010).

Pese a lo anterior, esta condición natural del ser humano no es suficiente. Tampoco es universal, homogénea, ni idéntica en todos, pues también se requiere una serie de condiciones culturales sociales e históricas que brinden soporte al desarrollo y construcción de los nuevos conocimientos, lo que hace de esta experiencia un proceso singular, único, variable y discontinuo, que inicia con la vida y termina con la muerte. Según esta escuela teórica, el sujeto aprende gracias a la interacción con sus pares o con un adulto experto (Rogoff, 1993).

Favorecer el desarrollo integral de los niños en el componente psicogenético y cultural y dentro de una propuesta de formación AVA requiere de principios didácticos en la educación infantil: integralidad, socialización, autonomía, paidocentrismo, globalización, vivencialidad, libertad, creatividad y lúdica. Para el interés específico de esta investigación, es relevante profundizar en este último, pues las experiencias formativas diseñadas centradas en la lúdica hacen del aprendizaje una experiencia de goce y disfrute, donde se manifiesta la libertad, la creatividad y la imaginación, características que lo convierten en un elemento fundamental para la construcción del ser humano como sujeto social y cultural.

En coherencia con el soporte teórico y la respectiva problemática, el objetivo de esta investigación es diseñar una propuesta pedagógica inter- 
disciplinar basada en AVA para la formación de las infancias ante los retos del posconflicto. Para ello se propuso llevar a cabo una investigación de tipo cualitativo con método descriptivo.

\section{Metodología}

El enfoque cualitativo de la investigación permite "observar fenómenos tal como se dan en su contexto natural, para posteriormente analizarlos" (Hernández, Fernández y Baptista, 2014, p. 149). En este sentido, se logra conocer en profundidad los factores socioeconómicos, de acceso y conocimiento del manejo a medios tecnológicos de los diferentes actores involucrados en el proyecto y se pueden identificar las necesidades de formación de los niños de Nimaima, para promover acciones educativas que, ante los retos del posconflicto, estén enfocadas en su formación sobre la base de la participación, la igualdad y el desarrollo social, ligado esto último a la potenciación de procesos de alfabetización mediática y digital. En cuanto al método seleccionado, se escogió uno descriptivo, pues, en palabras de Hernández et al., "los estudios descriptivos buscan especificar las propiedades, las características y los perfiles de las personas, grupos, comunidades, procesos, objetivos o cualquier otro fenómeno que se someta a un análisis" (2014, p. 80). Este método recurre a la descripción e interpretación para comprender la realidad social en la que están inmersos los niños vinculados al proyecto. El interés principal de la investigación está en función de lograr reconstruir las condiciones contextuales para configurar una fuente de información del territorio desde la cual realizar el diseño y validación de una propuesta pedagógica pertinente, que responda de manera directa a las necesidades formativas del territorio en donde se lleva a cabo el proyecto

La investigación parte de la caracterización de la comunidad educativa y de recoger información que permita construir una propuesta pedagógica situada a partir del modelo instruccional ADDIE y fundamentada en la didáctica de la educación infantil. Para garantizar que este diseño cumpla con las condiones técnicas y pedagógicas en coherencia con la caraterización del población, se realizó una prueba instrumental que involucró a maestros, mediante la aplicación de un cuestionario tipo Liker. Basado la indagación perceptual de los docentes, este permite validar el ambiente de aprendizaje y corroborar la usabilidad y calidad pedagógica de las herramientas informacionales en cuanto a los factores técnicos, gráficos y pedagógicos de la propuesta de formación.

\section{Resultados}

La caracterización de la población indica que esta es predominantemente rural y ha sido víctima del conflicto armado. En cuanto al proceso de diseño de la propuesta pedagógica, en un primer momento se trató de un aula virtual de aprendizaje en la plataforma Moodle y, en una segunda instancia, derivó en una adaptación a una aplicación offline. Adicionalmente, la validación por parte de los docentes dio valor a los aspectos técnicos y los factores estéticos y de funcionalidad del ambiente de aprendizaje. En la caracterización de los niños y sus familias se extrajo información sociodemográfica y las condiciones del proceso de paz y posconflicto.

Con respecto a la información socioeconómica de las familias del municipio de Nimaima involucradas en el proyecto, se puede afirmar que los padres y madres son adultos jóvenes con edades comprendidas entre 31 a 40 años, de procedencia rural, dispersos en diferentes veredas, pero ubicados relativamente cerca del colegio. En este contexto rural se presentan formas particulares de uso del espacio y relaciones sociales determinadas por la interacción con la naturaleza y la armonía con los demás pobladores. Lo rural, en Colombia, refiere a la vida en el campo, entendida como sinónimo de dilación, de tradición, de localismo (Mejía y Zuluaga, 2004). En el mismo sentido, en su mayoría, las familias están con- 
formadas por padres, madres e hijos, con lo cual se identifica una tradición familiar nuclear. La mayoría de las personas se ocupan en el sector agrícola y en el hogar: como se mencionó, ostentan bajos niveles de formación y poco interés por las innovaciones y avances tecnológicos; no obstante, cuentan con dispositivos móviles. Acerca del proceso de paz y el posconflicto, se reitera que Nimaima está catalogado como uno de los sectores nacionales más afectados por la confrontación armada. Entre los hechos victimizantes que ha sufrido la población se encuentran homicidios, desplazamiento, desplazamiento forzado, tortura, amenazas, desaparición forzada y maltrato físico y psicológico.

Por su parte, los resultados de la propuesta pedagógica confirman los hallazgos respecto a su perspectiva teórica y didáctica, los propósitos de formación, las actividades, la evaluación y la mediación tecnológica utilizada para llegar a los estudiantes en un contexto de posconflicto. Con respecto a la mediación tecnológica, es importante aclarar que, como resultado de la caracterización de las familas y los niños, donde se da un uso cotidiano del celular en los hogares, el AVA diseñado inicialmente en la plataforma Moodle se transformó en una aplicación offline. Se tomó esta decisión debido a que la conexión a internet en las instituciones educativas es insuficiente y su recurso tecnológico computacional se reduce al uso de tabletas que funcionan con el sistema operativo Android. Esta condición exigió diseñar una aplicación que funcionara en dispositivos móviles sin conexión a la red.

Esta propuesta pedagógica, a propósito de la caracterización que permite identificar los bajos niveles escolares de las familias e inferir su poca participación en las dinámicas escolares de los niños, exige fortalecer en los estudiantes las competencias mediáticas. Como se dijo, estas hacen referencia al dominio de conocimientos, habilidades y actitudes relacionados con el manejo de la tecnología, los procesos de interacción, la producción y la difusión y expresión estética de mensajes. Respecto de cada dimensión se diseñaron indicadores relacionados con el ámbito de participación de los niños, quienes constantemente reciben mensajes, interactúan con ellos (ámbito del análisis) y también los producen (ámbito de la expresión). La Tabla 1 presenta las dimensiones y sus indicadores.

De acuerdo con lo anterior, la propuesta pedagógica se basa en los principios didácticos de la educación infantil y está organizada en cuatro pasos fundamentados en las dimensiones e indicadores de la educación mediática y en los lineamientos gubernamentales para el trabajo en los territorios víctimas del conflicto.

La propuesta pedagógica se activó, en primer término, como un AVA en la plataforma Moodle, en cuyo diseño se recurrió a términos gráficos intuitivos y atractivos visualmente, valiéndose principalmente de la imagen y el color.

Habida cuenta de que la interactividad y la usabilidad en los AVA constituyen aspectos fundamentales del diseño de la interfaz, como elementos de comunicación e interacción con el estudiante, se enmarcó el diseño en la propuesta pedagógica y la ludomática. Con esto último se ofreció una ruta o secuencia para la realización de las actividades que se propusieron, a manera de juego. Estas actividades están diseñadas para que el estudiante "juegue" haciendo uso de aplicaciones o programas (Jigsaw, Grabadora de Audio, Sumopaint), lo que a su vez requiere tutoriales que orienten su uso.

Durante la implementación del aula virtual se evidenció la mencionada falta de conectividad a internet, limitante para el desarrollo del AVA; además, se estableció que no se cuenta con computadores suficientes para la implementación. Por ello, el equipo de investigación se dio a la tarea de diseñar un aplicativo móvil como un recurso educativo abierto (Zamora et al., 2017) de funcionamiento offline, que la comunidad educativa pudiera utilizar sin restricciones de conectividad. 
Tabla 1. Dimensiones e indicadores de la educación mediática

\begin{tabular}{|c|c|c|c|c|}
\hline Ámbitos & Tecnología & $\begin{array}{l}\text { Procesos de } \\
\text { interacción }\end{array}$ & $\begin{array}{l}\text { Procesos de } \\
\text { producción y difusión }\end{array}$ & Estética \\
\hline Análisis & $\begin{array}{l}\text { Comprensión del papel que } \\
\text { desempeñan TIC en la sociedad y } \\
\text { sus posibles efectos. } \\
\text { Habilidad para interactuar de } \\
\text { manera significativa con medios } \\
\text { que permiten expandir las } \\
\text { capacidades mentales. }\end{array}$ & $\begin{array}{l}\text { Capacidad de } \\
\text { gestionar el } \\
\text { ocio mediático, } \\
\text { convirtiéndolo en } \\
\text { oportunidad para el } \\
\text { aprendizaje. }\end{array}$ & $\begin{array}{c}\text { Conocimiento de las } \\
\text { diferencias básicas } \\
\text { entre producciones } \\
\text { individuales y } \\
\text { colectivas. }\end{array}$ & $\begin{array}{c}\text { Capacidad de extraer } \\
\text { placer de los aspectos } \\
\text { formales, es decir, } \\
\text { no solo de lo que se } \\
\text { comunica, sino también } \\
\text { de la manera como se } \\
\text { comunica. }\end{array}$ \\
\hline Expresión & $\begin{array}{c}\text { Capacidad de adecuar las } \\
\text { herramientas tecnológicas a los } \\
\text { objetivos comunicativos que se } \\
\text { persiguen. } \\
\text { Capacidad de elaborar y } \\
\text { manipular imágenes y sonidos } \\
\text { con conciencia de cómo se } \\
\text { construyen las representaciones } \\
\text { de la realidad. }\end{array}$ & $\begin{array}{l}\text { Capacidad de } \\
\text { llevar a cabo un } \\
\text { trabajo colaborativo } \\
\text { mediante la } \\
\text { conectividady } \\
\text { la creación de } \\
\text { plataformas que } \\
\text { facilitan las redes } \\
\text { sociales. }\end{array}$ & $\begin{array}{l}\text { Capacidad de } \\
\text { seleccionar mensajes } \\
\text { significativos, de } \\
\text { apropiarse de ellos } \\
\text { y de transformarlos } \\
\text { para producir nuevos } \\
\text { significados. }\end{array}$ & $\begin{array}{l}\text { Capacidad de producir } \\
\text { mensajes elementales } \\
\text { que sean comprensibles } \\
\text { y contribuyan a } \\
\text { incrementar los } \\
\text { niveles personales o } \\
\text { colectivos de creatividad, } \\
\text { originalidad y } \\
\text { sensibilidad. }\end{array}$ \\
\hline
\end{tabular}

Fuente: elaboración propia.

Tabla 2. Momentos de la propuesta pedagógica

\begin{tabular}{|c|c|c|c|}
\hline Momento & Antes & Durante & Al finalizar \\
\hline 1 & $\begin{array}{l}\text { Solicitar a los estudiantes } \\
\text { llevar fotos de lugares o } \\
\text { escenarios representativos o } \\
\text { favoritos (o que consideren } \\
\text { significativos) de su } \\
\text { territorio, haciendo uso de la } \\
\quad \text { aplicación. }\end{array}$ & $\begin{array}{l}\text { Socializar las fotografías recolectadas por } \\
\text { los estudiantes, solicitándoles una breve } \\
\text { explicación sobre lo que motivó a tomarla. } \\
\text { Dialogar sobre los escenarios fotografiados, } \\
\text { intentando indagar sobre el presente, el } \\
\text { pasado y el posible futuro. }\end{array}$ & $\begin{array}{l}\text { Solicitar a los estudiantes como } \\
\text { tarea un audio o video donde un } \\
\text { abuelo, profesor, vecino, entre } \\
\text { otros, cuente sobre ese lugar } \\
\text { fotografiado. El audio o video } \\
\text { debe crearse con la aplicación. }\end{array}$ \\
\hline 2 & $\begin{array}{l}\text { Traer la entrevista grabada y } \\
\text { la foto impresa. }\end{array}$ & $\begin{array}{l}\text { Pegar las fotos en el salón, a modo de galería. } \\
\text { Escuchar las grabaciones de audio para } \\
\text { identificar a qué foto hacen referencia. } \\
\text { Generar un diálogo entre docente y } \\
\text { estudiantes acerca de la experiencia, e indagar } \\
\text { acerca de la percepción que estos últimos } \\
\text { tienen de su territorio. }\end{array}$ & $\begin{array}{l}\text { Realizar un dibujo que recoja } \\
\text { la percepción del territorio, } \\
\text { configurada a partir de las fotos, } \\
\text { los audios y el diálogo en clase. }\end{array}$ \\
\hline 3 & $\begin{array}{l}\text { Traer el dibujo del territorio } \\
\text { en una cartulina de } 50 \times 35 \\
\text { cm, utilizando diferentes } \\
\text { materiales reciclables. }\end{array}$ & $\begin{array}{l}\text { Hacer la exposición de los dibujos resaltando } \\
\text { los aspectos positivos. }\end{array}$ & $\begin{array}{c}\text { El profesor, con el mapa del } \\
\text { municipio, ubica los dibujos de } \\
\text { los estudiantes para aportar a } \\
\text { la resignificación del territorio y } \\
\text { resaltar lo positivo. }\end{array}$ \\
\hline 4 & $\begin{array}{l}\text { El docente hace una } \\
\text { indagación en las diferentes } \\
\text { entidades municipales, con } \\
\text { el fin de recoger información } \\
\text { que permita complementar } \\
\text { el mapa construido con los } \\
\text { estudiantes. }\end{array}$ & $\begin{array}{c}\text { El docente amplía el mapa con respecto a } \\
\text { la información recopilada, intentando que } \\
\text { el estudiante reconfigure su comprensión } \\
\text { acerca del territorio en tanto punto de partida } \\
\text { para visibilizarse como un protagonista en su } \\
\text { transformación. }\end{array}$ & $\begin{array}{c}\text { Entrega oficial del mapa al } \\
\text { colegio para que se exponga en } \\
\text { una zona pública y se socialice } \\
\text { en un evento institucional con la } \\
\text { comunidad educativa. }\end{array}$ \\
\hline
\end{tabular}

Fuente: elaboración propia. 
Figura 1. Portada del AVA de Ludomática

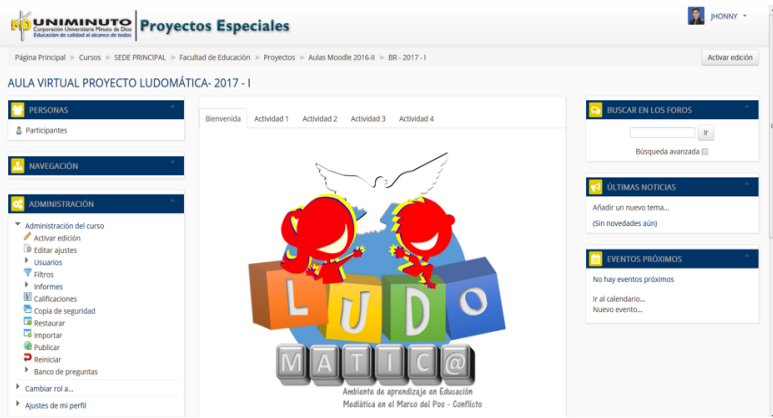

Fuente: Ambiente Virtual de Aprendizaje diseñado con la herramienta Moodle.

\section{Figura 2. Mapa de actividades en el aula virtual}

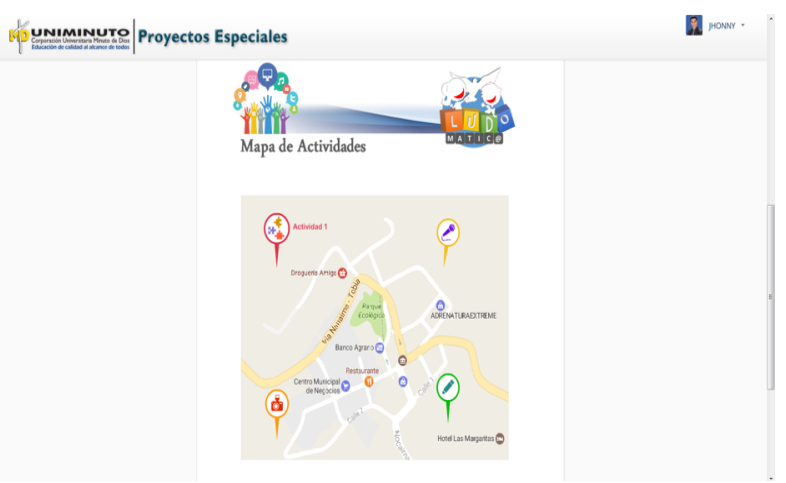

Fuente: Ambiente Virtual de Aprendizaje diseñado con la herramienta Moodle.

La aplicación también se desarrolló con el diseño instruccional ADDIE, empleado para el AVA anterior. Se utilizó la misma estructura pedagógica y se conservó la esencia lúdica del uso de las herramientas TIC de uso libre que cumplieran con las mismas posibilidades de trabajo de la web. Cada actividad cuenta con las instrucciones y las herramientas tecnológicas para desarrollarla. Respecto del apartado gráfico, se ilustró un escenario atractivo para la población infantil; respecto de la usabilidad, se tuvo especial cuidado de que la funcionalidad del aplicativo permitiera una navegación simple, conducente al desarrollo de competencias mediáticas en la comunidad educativa.

\section{Figura 3. Pantallas de presentación de la aplicación Ludomática}

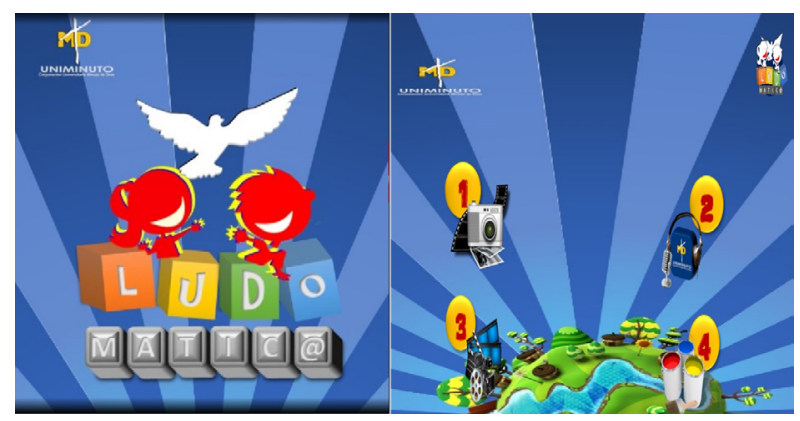

Fuente: Aplicación móvil diseñada en APPinventor

El resultado de la validación fue formulado para evaluar la calidad de los factores pedagógicos, técnicos, de diseño y funcionalidad de la aplicación educativa móvil Ludomática, pensada para mejorar las habilidades comunicativas y tecnológicas en las instituciones de educación primaria del municipio de Nimaima.

Para obtener los resultados se realizaron encuestas tipo Likert a los docentes, las cuales se analizaron con métodos estadísticos de tendencia central. Con esto se buscó evaluar los diferentes aspectos del ambiente que garantizaran pertinencia y calidad. Este mismo ejercicio tuvo por objeto que cada uno de los maestros contara con los conocimientos técnicos y pedagógicos durante la implementación de la estrategia en cada una de las escuelas del municipio estudiado. Respecto del uso del software SPSS para el respectivo análisis de los datos, concordamos con Hefetz, Liberman y Salas, quienes señalan que "el uso de un paquete estadístico para la interpretación de datos permite introducir un elemento de confiabilidad adicional a los resultados de la investigación, además [que] arroja información contundente que al momento de ser analizada aporta a la solución del problema y cumplimiento del objetivo" (2017, p. 395). 
Figura 4. Evaluación y formación pedagógica del ambiente de aprendizaje con los maestros del municipio de Nimaima

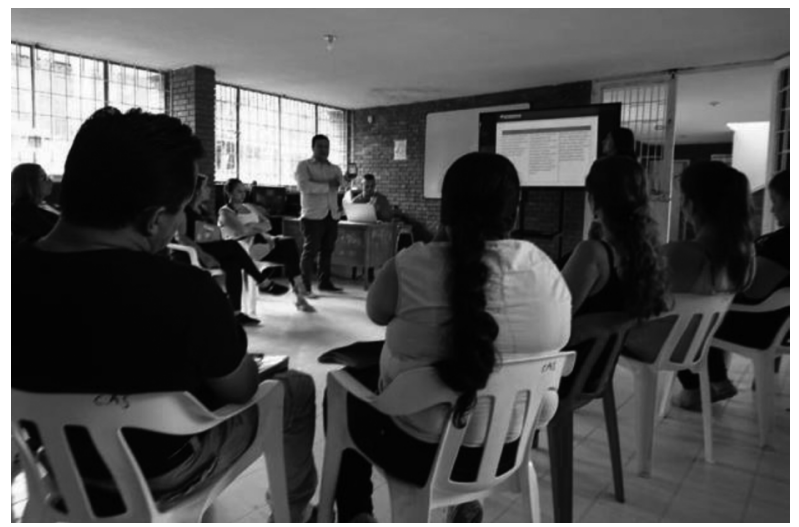

Fuente: elaboración propia.

Este instrumento tomó como referente la Evaluación de la Calidad de los Cursos Virtuales de la Universidad Nacional de Educación a Distancia de España (UNED) y el trabajo "Evaluación de las aplicaciones multimedia: Criterios de calidad de la Unidad de Tecnología Educativa" de la Universidad de Valencia. Se adaptaron los factores categóricos en función de los propósitos del proyecto de investigación "Ambientes de aprendizaje desde la ludomática para el desarrollo de la comunicación y la tecnología de los niños en el marco del posconflicto". Adicionalmente, su aplicación se realizó a partir de una muestra censal llevada a cabo con los nueve maestros de las escuelas rurales que carecen de conexión a internet. En este sentido, se diseñó la aplicación para que fuese utilizada por la comunidad educativa en las tabletas digitales y teléfonos móviles de los padres de familia.

El instrumento se diseñó para evaluar en cuatro categorías: los términos técnicos, lo estético, lo pedagógico y la funcionalidad de la herramienta. Respecto de la consistencia interna, muestra un nivel sobresaliente de confiabilidad: su verificación por el método de alfa de Cronbach arrojó un resultado de o,9420. A continuación, se presentan los resultados estadísticos de las categorías de evaluación de la calidad del ambiente de aprendizaje.

\section{Figura 5. Aspectos técnicos del ambiente de aprendizaje}

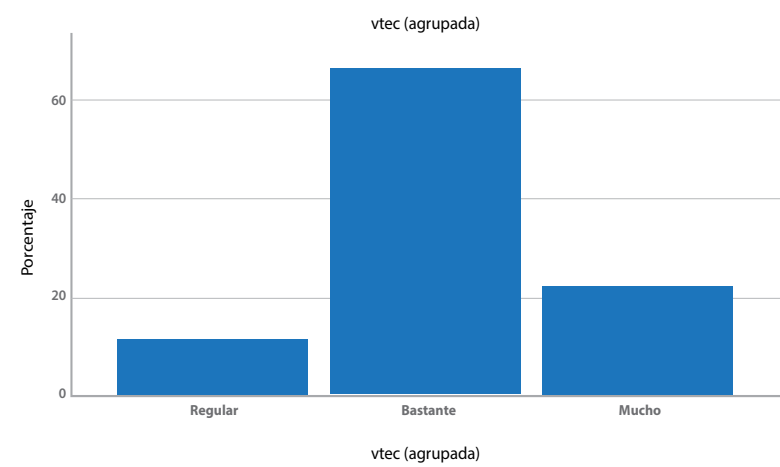

Fuente: elaboración propia.

Respecto del funcionamiento técnico del ambiente de aprendizaje, se concluye que $66 \%$ de nivel de desarrollo técnico es bastante satisfactorio, a excepción de la velocidad de procesamiento y presentación de la información, lo cual se constituye un factor a mejorar. Esto requiere una inversión en elementos de ingeniería que puedan ser publicados para libre descarga.

\section{Figura 6. Factores estéticos del ambiente}

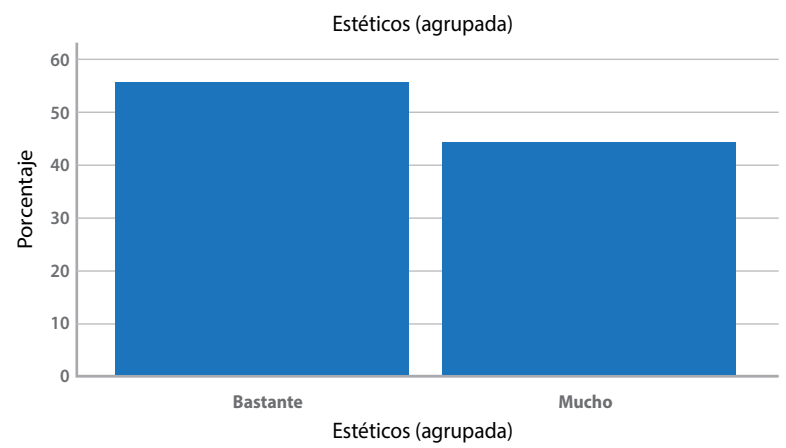

Fuente: elaboración propia.

En lo que atañe a las cuestiones estéticas, el ambiente de aprendizaje se mueve entre los dos indicadores superiores, con una moda de 5 , que es el factor más alto. Ello demuestra que la presentación y adecuación gráfica es satisfactoria y agradable para su uso por parte de la comunidad. 


\section{Figura 7. Factores asociados al aprendizaje del ambiente}

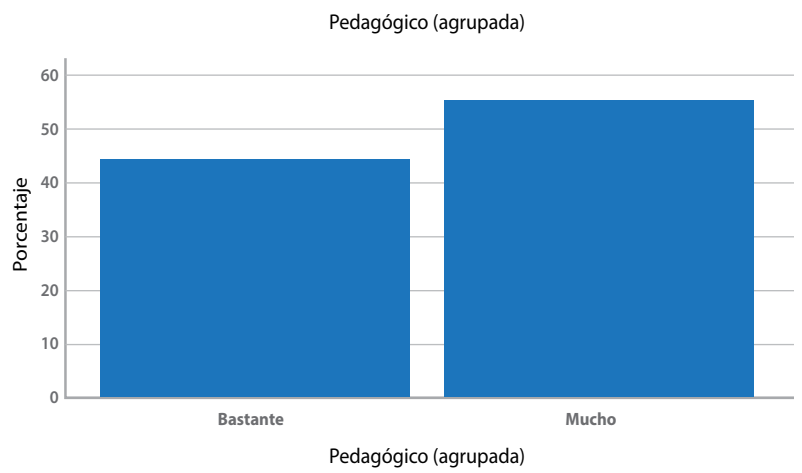

Fuente: elaboración propia.

Los factores pedagógicos y su funcionalidad para el aprendizaje, que son parte esencial de esta investigación, presentan un resultado satisfactorio: tras la implementación y su mediación por parte de profesionales de la educación, la moda estadística asciende a 5 y los valores recurrentes oscilan entre los dos factores más altos en nivel de calidad. Se rescata que la herramienta tiene diversas posibilidades de uso pedagógico como una mediación tecnológica para el aprendizaje, puesto que respeta diferentes ritmos y permite la integración de la comunidad en el proceso de aprendizaje de los niños.

\section{Figura 8. Factores de funcionalidad del ambiente}

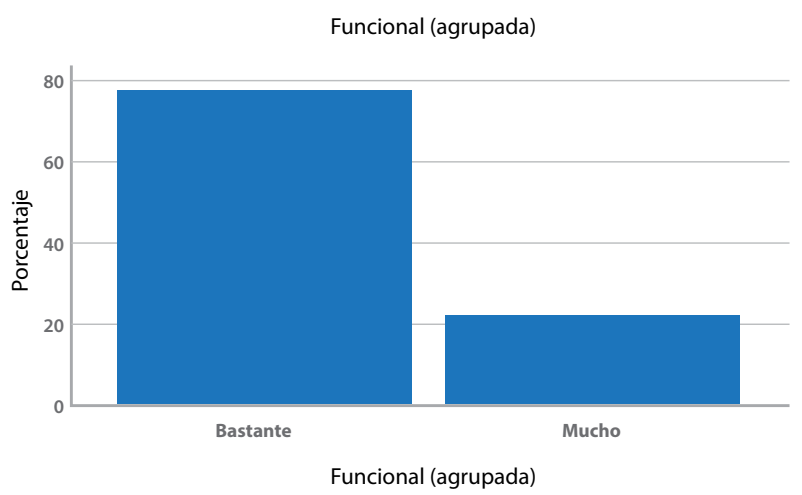

Fuente: elaboración propia.
Finalmente, en lo referido a la funcionalidad, se obtuvo una moda estadística de 4 , lo que muestra que la herramienta cuenta con características de eficacia óptimas para el logro de los factores de aprendizaje. Cabe destacar, además, que la relación inversión-eficacia es muy favorable, por cuanto la herramienta ha sido desarrollada mediante software libre y está disponible para ser descargada de forma gratuita.

\section{Discusión y conclusiones}

Los hallazgos de esta investigación permiten identificar que el diseño de una propuesta pedagógica interdisciplinar basada en ambientes virtuales de aprendizaje para la formación de las infancias ante los retos del posconflicto -para el caso estudiado, en la población de Nimaima- aporta a la disminución de brechas digitales propias de poblaciones rurales como esta. Los resultados de la primera parte de la investigación permiten evidenciar la baja conectividad y el acceso limitado a herramientas tecnológicas, lo cual afecta directamente los procesos de enseñanza-aprendizaje en los niños, especialmente si se toman en cuenta las exigencias de la sociedad del conocimiento actual. Esta situación se asemeja a lo que reportan Förster y Rojas-Barahona (2014), cuyos resultados de su investigación encontraron que el desarrollo de habilidades de alfabetización en la población infantil de zonas rurales de Chile es significativamente menor al de esta misma en zonas urbanas. De allí la importancia de diseñar programas de formación para las infancias rurales, que apoyen los procesos formativos adelantados dentro de sus familias y en las prácticas cotidianas de convivencia de la zona.

En este sentido, diseñar una propuesta pedagógica basada en una APP offline, como consecuencia de la carencia de herramientas tecnológicas y de conectividad en las familias y en la institucion, representa un aporte significativo, ante la necesidad de disminuir la brecha digital y las carencias en la formación propias de zonas rurales en contexto de 
posconflicto, como es el caso de Nimaima. Así, entonces, esta es una contribución a la alfabetización digital, por cuanto, además de reducirse la brecha señalada, se posibilita un acercamiento seguro y responsable a las TIC e internet (Fernández, Peñalva, Irazabal y López, 2017).

Sumado a lo anterior, esta estrategia favorece el acercamiento y uso de las herramientas tecnológcas y, para el caso de esta investigación, aporta la formación de las infancias desde la perspectiva de la educación mediática. En este sentido, los recursos tecnológicos, como Moodle, los dispositivos móviles y la aplicación offline son "una herramienta, no un fin en sí mismas" (Cabañero, 2016, p. 585); por lo tanto, se conciben esenciales en la medida en que contribuyen a la resignificación del territorio y a la reconstrucción de tejido social. De acuerdo con esto, es importante resaltar que el uso de las herramientas tecnológicas en los procesos de enseñanza-aprendizaje requiere una planeación que busque la transformación de la persona y de la sociedad: en el caso que nos ocupa, por ejemplo, el uso de las herramientas tecnológicas pretende que el estudiante realice una serie de actividades que le demandan transitar por el territorio, dialogar con los otros y participar activamente en la toma de decisiones, entre otras tareas.

Asimismo, el uso de recursos tecnológicos en el marco del proyecto descrito, entre los que se cuentan el teléfono móvil y la aplicación offline, contribuye a los procesos democráticos de diálogo y participación, pero no por el uso de las herramientas tecnológicas en sí, sino por el papel que estas desempeñan: su carácter primordial en la investigación viene de que están concebidas para producir encuentros participativos entre estudiantes, profesores, padres de familia y otras personas de la comunidad. Igualmente, la propuesta se centra en la participación de los estudiantes, porque las actividades están diseñadas con esta lógica y con ello se corrobora que "es posible implementar una cultura de la participación en la que el alumnado pueda expresarse a través de los medios, crear y publicar contenidos", así como contribuir a la transformación de los procesos comunicativos en el salón de clase a partir de "la creación de espacios de encuentro y reflexión y también de negociación y organización entre el alumnado y con el docente" (Hergueta, 2017, pp. 236-237).

Uno de los propósitos de esta investigación radicó en generar experiencias educativas con las cuales se avance en perspectiva del posconflicto, porque, como se enunció, los procesos educativos son fundamentales para agenciar cambios en las prácticas socioculturales de quienes se han visto afectados por la violencia. En ese contexto, el diseño de esta propuesta se anticipa a las patologías del posconflicto descritas por Piris (2010), cuyos alcances en la propuesta pedagógica se describen a continuación:

- $\quad$ Reorganización territorial: la propuesta busca que el estudiante resignifique su territorio y encuentre en él otras posibilidades para habitarloy transformarlo en favor de la comunidad.

- Paso sociocultural de la fuerza al consenso y negociación: la propuesta se centra en fortalecer los procesos de participación y diálogo entre los actores de la comunidad educativa.

- Necesidad de formación ante las dinámicas propias del posconflicto: para atenderla se generan estrategias metodológicas y actividades de la propuesta que fortalecen el aprendizaje colaborativo a través de la creación de comunidades de aprendizaje y uso de las TIC.

Asimismo, con la ejecución de esta investigación se puede corroborar que es posible avanzar en prácticas educativas que aporten a la construcción de paz, ya que "la consecución o el trabajo por la paz va más allá de la denuncia de la guerra" (Vinyamata, 2015, p. 21). Por lo anterior, se considera que el trabajo con los niños es un paso importante para construir una cultura de paz, pues su condición de infantes les concede más apertura a las transformaciones culturales y, además, porque son ellos quienes construyen el presente y, dependiendo de ello, habitarán el futuro. 
Gracias al procedimiento de análisis realizado, es posible afirmar que existe una gran receptividad y apoyo por parte de los docentes ante el diseño y aplicación de la propuesta de formación. Esto resulta fundamental, por cuanto en sus prácticas cotidianas yace la posibilidad de trascender del diseño para llegar a una aplicación articulada con las necesidades y compromisos de formación que exige la institución educativa. Ahora bien, esta investigación no desconoce las apreciaciones de Cabañero al respecto, cuando dice:

Los profesores son el principal motor de cambio en el mundo educativo. Sin embargo, parece que su formación inicial y continua no les dota de las actitudes y estrategias para ello. De esta ma- nera, pensamos que, en muchos casos, las carencias de medios tecnológicos puede ser más una excusa que una dificultad real para conseguir cambios metodológicos profundos y una integración de las TIC en la escuela. (2016, p. 585)

En este sentido, el proyecto de investigación realizado ofrece a los docentes una propuesta de formación para los niños de Nimaima que, al estar soportada en la creación de un ambiente virtual de aprendizaje lúdico, promueve la formación del maestro en el uso de la herramienta tecnológica y en la cualificación de su práctica, a través de la mejora y trasformación de la misma, como resultado de la implementación de nuevas estrategias de formación que maximizan el aprendizaje de sus estudiantes.

\section{Referencias}

Antoranz, E. y Villalba, J. (2010). Desarrollo cognitivo y motor. Madrid: Editex.

Belloch, C. (2013). Diseño instruccional. Valencia: Unidad de Tecnología Educativa (UTE), Universidad de Valencia. Recuperado de https://www.uv.es/ bellochc/pedagogia/EVA4.pdf

Berdal, M. (2008). The UN Peacebuilding Commission: The rise and fall of a good idea. En Pugh, M., Cooper, N. y Turner, M. (eds.), Whose peace? Critical perspectives on the political economy of peacebuilding (pp. 356-372). Nueva York: Palgrave Macmillan.

Berdal, M. y Malone, D. (2000). Greed and grievance: Economic agendas in civil wars. Nueva York: Lynne Rienner, IDRC.

Cabañero, J. (2016). La incorporación de las tecnologías de la información y la comunicación y el papel del profesorado de educación infantil y primaria. Estudio comparativo entre West Palm Beach County y la Comunidad de Madrid. Tesis de Doctorado en Educación, Universidad Complutense de Madrid, España.

Collier, P. et al. (2003). Breaking the conflict trap: Civil war and development policy. Washington: Banco Mundial, Oxford University Press.

Daskal, E. (2018). Let's be careful out there...: How digital rights advocates educate citizens in the digital age. Information Communication and Society, 21(2), 241-256. DOI: https://doi.org/10.1080/1369118X.2016.1271903

DNP - Departamento Nacional de Planeación (2018). Sisbén. Reporte de la Base Nacional Certificada. Recuperado de https://www.sisben.gov.co/Territorios/Paginas/Reportes\%20Base\%20Certificada/ano2018.aspx 
Jiménez Dato, A., De Currea-Lugo, V.y Grabe Loewenherz, V. (2008). Educación en Emergencia. Madrid: Instituto de Estudios sobre Conflictos y Acción Humanitaria.

Fernández-Montalvo, J., Peñalva, A., Irazabal, I. y López-Goñi, J. J. (2017). Efectividad de un programa de alfabetización digital para estudiantes de educación primaria. Cultura y Educación, 29(1), 1-30. DOI: https://doi.or g/10.1080/11356405.2016.1269501

Ferrés, J. y Piscitelli, A. (2012). La competencia mediática: propuesta articulada de dimensiones e indicadores. Comunicar, 38, 75-82. DOI: https://doi.org/10.3916/C38-2012-02-08

Filipov, F. (2008). Post-conflict peacebuilding: Strategies and lessons from Bosnia and Herzegovina, El Salvador and Sierra Leone. Some thoughts from the rights to education and health. Santiago de Chile: Organización de Naciones Unidas.

Förster, C. y Rojas-Barahona, C. (2014). Niños preescolares vulnerables de sectores rurales: importancia de las prácticas del hogar y la asistencia a jardín infantil en el desarrollo de habilidades de alfabetización temprana. Cultura y Educación, 26(1), 476-504. DOI: https://doi.org/10.1080/11356405.2014.973668

Fride - Fundación para las Relaciones Internacionales y el Diálogo Exterior - (2006). El posconflicto bajo el prisma de las víctimas. Recuperado de http://fride.org/descarga/BGR_Posconflic_ESP_dico6.pdf

Garzón, J. (2003). El posconflicto en Colombia. Coordenadas para la paz. Tesis de pregrado en Derecho, Pontificia Universidad Javeriana, Bogotá, Colombia. Recuperado de http://www.javeriana.edu.co/biblos/tesis/derecho/dere5/TESIS47.pdf

Hefetz, A., Liberman, G. y Salas, N. (2017). Ideas sobre modelos estadísticos avanzados: un desafío para la investigación en cultura y educación. Cultura y Educación, 29(1), 395-408. DOl: https://doi.org/10.1080/1135640 5.2017 .1368163

Hergueta, E. (2017). Educación mediática. Propuesta metodológica para el desarrollo de la competencia mediática en la práctica educativa. Tesis doctoral, Universidad Nacional de Educación a Distancia.

Hernández, R., Fernández, C.y Baptista, P. (2014). Metodología de la investigación. México: McGraw-Hill Interamericana.

Herrera, M. (2006). Consideraciones para el diseño didáctico de ambientes virtuales de aprendizaje: una propuesta basada en las funciones cognitivas del aprendizaje. Revista Iberoamericana de Educación, 5(38), 1-19. Recuperado de https://www.researchgate.net/publication/28108445_Consideraciones_para_el_diseno_didactico_de_ambientes_virtuales_de_aprendizaje_una_propuesta_basada_en_las_funciones_ cognitivas_del_aprendizaje

Infante, A. (2011). El papel de la educación en situaciones de posconflicto: estrategias y recomendaciones. Revista Hallazgos, $11(21), 223-245$.

Lillard, A. S. et al. (2017). Montessori preschool elevates and equalizes child outcomes: A longitudinal study. Frontiers in Psychology, 8(10), 1-19. DOI: https://doi.org/10.3389/fpsyg.2017.01783 
ISSN 0123-1294 | e-ISSN 2027-5358 | Educ.Educ. Vol. 22. No.3 | Septiembre-diciembre de 2019 | pp.359-376.

Universidad de La Sabana | Facultad de Educación

Mejía, H. y Zuluaga, P. (2004). Lo rural y lo campesino. Foro virtual sobre educación y desarrollo rural. Medellín: Corporación La Ceiba.

Morin, E. (2009). Introducción al pensamiento complejo. España: Gedisa.

Nicolai, S. y Triplehorn, C. (2003). The role of education in protecting children in conflict. Londres: Humanitarian Practice Network.

Pérez, P. (2014). Colombia: de la educación en emergencia hacia una educación para el posconflicto y la paz. Revista Interamericana de Investigación, Educación y Pedagogía, 7(2). DOI: https://doi.org/10.15332/s1657107X.2014.0002.06

Piris, A. (2010). Bases de la rehabilitación posbélica. En Osorio, T. (coord.), Después de la guerra: un manual para la reconstrucción posbélica, con casos de estudio sobre Guatemala, El Salvador, Bosnia, Mozambique, el papel de la UE y el enfoque de género en la rehabilitación del posconflicto (pp.13-104). Barcelona: Icaria.

Pounaki, E., Givi, M. y Fahimnia, F. (2017). Investigate the relation between media literacy and information literacy of students of communication science and knowledge and information science. Iranian Journal of Information Processing Management, 32(2), 581-604. Recuperado de http://jipm.irandoc.ac.ir/browse. php?a_code $=$ A-10-3339-1\&slc_lang $=$ en \&sid $=1$

Rogoff, B. (1993). Aprendices del pensamiento. Barcelona: Paidós.

Salinas, J. (2004). Innovación docente y uso de las TIC en la enseñanza universitaria. Revista Universidad y Sociedad del Conocimiento, 1(1), 1-16. DOI: http://doi.org/10.7238/rusc.v1i1.228

Sandberg A. et al. (2017). Children's perspective on learning: An international study in Denmark, Estonia, Germany and Sweden. Early Childhood Education Journal, 45(1), 71-81. DOI: https://doi.org/10.1007/s10643015-0759-5

Vinyamata, E. (2015). Conflictología. Revista de Paz y Conflictos, 8(1), 9-24. Recuperado de http://www.redaly c. org/pdf/2050/205039638001.pdf

Zamora, R. et al. (2017). Implementación de un recurso educativo abierto a través del modelo del diseño universal para el aprendizaje teniendo en cuenta evaluación de competencias y las necesidades individuales de los estudiantes. Revista Espacios, 38(5), 1-3. Recuperado de http://www.revistaespacios.com/ a17v38no5/17380503.html 